\title{
AVALIAÇÃO DOS PARÂMETROS DE PROJETO DE MEDIDORES DE VAZÃO TIPO VENTURI UTILIZANDO FLUIDODINÂMICA COMPUTACIONAL
}

\author{
M. G. de PAULA SANTOS ${ }^{1}$, R. BÉTTEGA ${ }^{1}$ \\ ${ }^{1}$ Universidade Federal de São Carlos, Departamento de Engenharia Química \\ E-mail para contato: melinegabriele@hotmail.com
}

\begin{abstract}
RESUMO - O Tubo de Venturi é um componente amplamente utilizado para medir a vazão de fluidos escoando em tubulações. Neste trabalho objetivou-se, a partir de simulações de escoamento utilizando CFD, a avaliação da variação de parâmetros geométricos clássicos do tubo de Venturi buscando otimizar a perda de carga no equipamento. Utilizando o software Fluent 14.0 realizaram-se simulações de escoamento em modelos de tubos de venturi que sofreram variações de \pm 10 e $20 \%$ no ângulos divergente e/ou convergente, para seis velocidades diferentes. Os resultados indicaram que apenas o ângulo divergente deve ser diminuído para atingir quedas de pressão menores. Por outro lado, o aumento de ângulos, tanto convergente quanto divergente mostrou-se, na maioria dos casos, uma boa alternativa.
\end{abstract}

\section{INTRODUÇÃO}

Fluidodinâmica computacional ou, em inglês, Computational Fluid Dynamics (CFD) é uma ferramenta de simulação numérica que pode ser aplicada a processos que envolvem escoamento de fluidos. O uso desse método possibilita simular as mais variadas geometrias e condições operacionais sem a necessidade da construção de protótipos, permitindo avaliar uma gama ampla de condições e geometrias.

Segundo a ABNT NBR Iso 5167-1, um tubo de Venturi é: "elemento que consiste em uma entrada convergente conectada a uma seção cilíndrica chamada 'garganta' e numa seção de expansão cônica, denominada 'divergente'.", sendo bastante utilizado para estudo de escoamento de fluidos por apresentar baixas perdas de carga. Guerra et al. (2012) estudaram o comportamento fluidodinâmico das fases gasosa e líquida na garganta de um tubo de venturi, variando a pressão e a fração volumétrica, tanto experimentalmente quanto através de simulações CFD. Eles puderam concluir que, para um mesmo gás ou vazão de líquido, o número de orifícios de injeção do fluido tem grande papel na dispersão do líquido, embora não exerça grande influência na queda de pressão.

Ainda na linha de simulação CF de tubos de Venturi, Honda et al. (2012), em outro estudo, realizaram simulações CFD de um escoamento bifásico ar-água a fim de avaliar numericamente a distribuição de líquido em um lavador venturi, comparando os resultados 


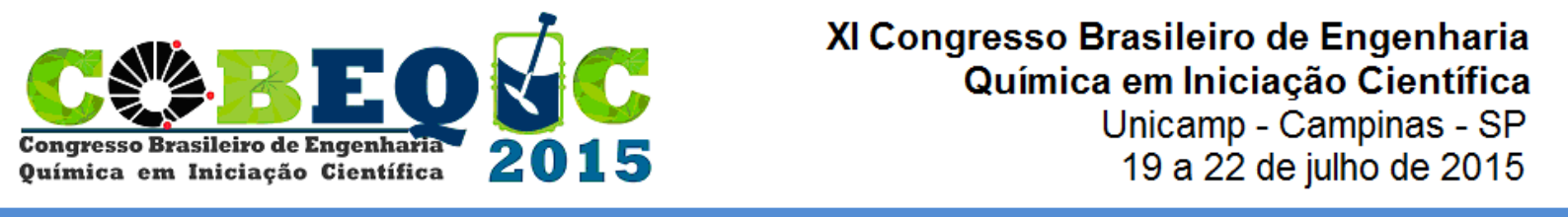

computacionais com os experimentais. Para variações no número de orifícios e vazão de ar e líquido foram obtidos bons resultados apenas para baixas vazões.

Considerando a facilidade da construção e avaliação de aspectos geométricos de equipamentos a partir de CFD, neste trabalho foi proposto estudar a configuração geométrica do tubo de Venturi e, a partir de modificações em sua estrutura, buscar uma configuração que oferecesse a menor perda de carga teórica.

\section{MATERIAIS E MÉTODOS}

\subsection{Materiais}

Para o desenvolvimento deste trabalho foram utilizados os seguintes materiais:

- $\quad$ Software ANSYS Gambit 2.4.6, para a geração da geometria e da malha computacional;

- $\quad$ Software ANSYS Fluent 14.0, para realizar as simulações numétricas

- $\quad$ Software Microsoft Excel 2010 para o tratametno do resultados;

- Microcomputador com processador Intel Celeron, 1,5 GHz, 1,44 GB RAM

\subsection{Metodologia}

Inicialmente, realizou-se uma série de testes para definir a configuração da malha a ser adotada nas simulações. Para isso, utilizando o software Gambit 2.4.6, criou-se diversas malhas, variando-se o espaçamento entre os nós. Este teste de malha indicou a configuração de malha mais adequada para a realização das simulações numéricas. Após análise dos testes de malha, definiu-se espaçamento entre nós de $0,5 \mathrm{~mm}$ a ser adotado em todas as malhas.

Na figura 1 é apresentado um esboço do equipamento considerado padrão para as simulações, bem como a malha adotada no trabalho é apresentada na figura 2.

Figura 1 - Dimensões do Tubo de Venturi Padrão.

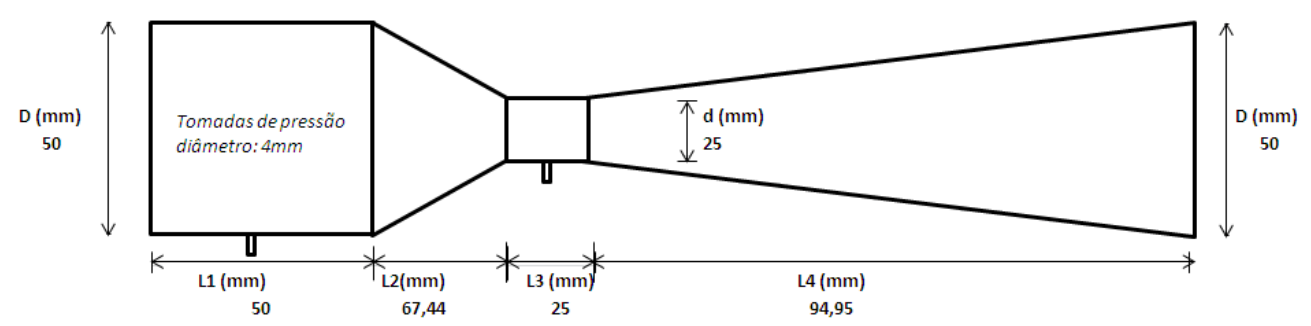


Figura 2 - Malha computacional

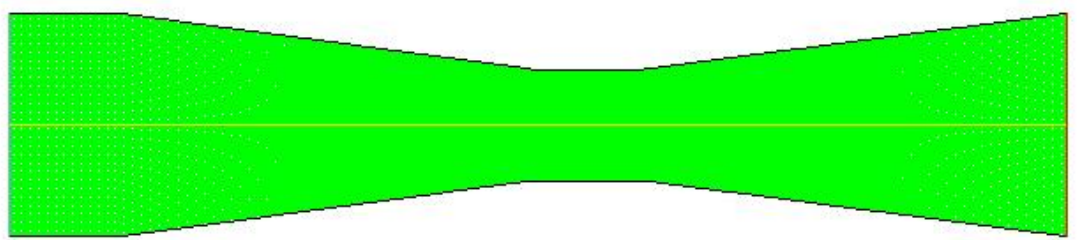

Na tabela 1 são apresentados os parâmetros adotados para todas as simulações realizadas neste trabalho:

Tabela 1. Parâmetros das Simulações

\begin{tabular}{|l|c|}
\hline Viscosidade: & Standard k-e \\
\hline Fluido: & $\begin{array}{c}\text { Ar - densidade: } 1,0 \mathrm{~kg} / \mathrm{m}^{3} \\
\text { viscosidade: } 2,09 \mathrm{e}-05 \mathrm{~kg} / \mathrm{m}-\mathrm{s}\end{array}$ \\
\hline Solution Methods: & $\begin{array}{c}\text { Pressão: standard } \\
\text { Momento: Second O.U. }\end{array}$ \\
\hline Solution Initialization: & $\begin{array}{c}\text { Turbulent Kinetic Energy: First O.U. } \\
\text { Turbulent Dissipation Rate: First O.U. }\end{array}$ \\
\hline Iterações: & Hybrid \\
\hline
\end{tabular}

Tabela 2 - Dimensões

\begin{tabular}{|c|c|c|c|c|c|c|c|c|c|c|c|c|c|}
\hline & V-1 & $\begin{array}{c}\mathrm{V}-2 \\
+20 \%\end{array}$ & $\begin{array}{c}V-3 \\
-20 \%\end{array}$ & $\begin{array}{c}\mathrm{V}-4 \\
+10 \%\end{array}$ & $\begin{array}{c}-5 \\
-10 \%\end{array}$ & $\begin{array}{c}V-6 \\
+20 \% c\end{array}$ & $\begin{array}{c}V-7 \\
-20 \% c\end{array}$ & $\begin{array}{c}\mathrm{V}-8 \\
+10 \% \mathrm{c}\end{array}$ & $\begin{array}{c}V-9 \\
-10 \% c\end{array}$ & $\begin{array}{c}V-10 \\
+20 \% d\end{array}$ & $\begin{array}{c}V-11 \\
-20 \% d\end{array}$ & $\begin{array}{c}\mathrm{V}-12 \\
+10 \% \mathrm{~d}\end{array}$ & $\begin{array}{c}-13 \\
-10 \% d\end{array}$ \\
\hline $\mathrm{D}(\mathrm{mm})$ & 50 & 50 & 50 & 50 & 50 & 50 & 50 & 50 & 50 & 50 & 50 & 50 & 50 \\
\hline Beta & 0,5 & 0,5 & 0,5 & 0,5 & 0,5 & 0,5 & 0,5 & 0,5 & 0,5 & 0,5 & 0,5 & 0,5 & 0,5 \\
\hline $\begin{array}{l}\text { ângulo } \\
\text { div. }\left({ }^{\circ}\right)\end{array}$ & 15 & 18 & 12 & 16,5 & 13,5 & 15 & 15 & 15 & 15 & 18 & 12 & 16,5 & 13,5 \\
\hline $\begin{array}{l}\text { ângulo } \\
\text { conv. }\left({ }^{\circ}\right)\end{array}$ & 21 & 25,2 & 16,8 & 23,1 & 18,9 & 25,2 & 16,8 & 23,1 & 18,9 & 21 & 21 & 21 & 21 \\
\hline $\mathrm{d}(\mathrm{mm})$ & 25 & 25 & 25 & 25 & 25 & 25 & 25 & 25 & 25 & 25 & 25 & 25 & 25 \\
\hline $\begin{array}{c}\mathrm{L} 1 \\
(\mathrm{~mm})\end{array}$ & 50 & 50 & 50 & 50 & 50 & 50 & 50 & 50 & 50 & 50 & 50 & 50 & 50 \\
\hline $\begin{array}{c}\mathrm{L} 2 \\
(\mathrm{~mm})\end{array}$ & 67,44 & 55,92 & 84,65 & 61,17 & 75,10 & 55,92 & 84,65 & 61,17 & 75,10 & 67,44 & 67,44 & 67,44 & 67,44 \\
\hline $\begin{array}{c}\mathrm{L} 3 \\
(\mathrm{~mm})\end{array}$ & 25 & 25 & 25 & 25 & 25 & 25 & 25 & 25 & 25 & 25 & 25 & 25 & 25 \\
\hline $\begin{array}{c}\text { L4 } \\
(\mathrm{mm})\end{array}$ & 94,95 & 78,92 & 118,93 & 86,21 & 105,61 & 94,95 & 94,95 & 94,95 & 94,95 & 78,92 & 118,93 & 86,21 & 105,61 \\
\hline $\begin{array}{l}\text { Comp } \\
(\mathrm{mm})\end{array}$ & 237,4 & 209,8 & 278,5 & 222,38 & 255,7 & 225,87 & 254,60 & 231,11 & 245,05 & 221,37 & 261,37 & 228,65 & 248,06 \\
\hline
\end{tabular}


Após a escolha da malha, foram feitas simulações utilizando os mesmos parâmetros dos testes de malha, para seis diferentes velocidades. Em seguida, variou-se os ângulos convergente e divergente em $\pm 10 \%$ e $\pm 20 \%$ (em relação ao modelo de venturi inicial ilustrado na figura 1), primeiro os dois ângulos ao mesmo tempo e em seguida cada ângulo isoladamente, resultando assim em treze tubos de venturi com dimensões diferentes. Na tabela 2 são apresentadas as geometrias dos venturis utilizados, considerando as variações nos parâmetros de projeto.

Efetuaram-se as simulações com as mesmas configurações iniciais (Tabela 2), para as mesmas velocidades do tubo-padrão. Além disso, foram construídos gráficos de velocidade e pressão estática para todas as simulações. A queda de pressão foi medida através da diferença de pressão entre dois pontos, sendo que a pressão estática é uma média de pressão na seção transversal, agindo igual em todas as direções.

\section{RESULTADOS E DISCUSSÃO}

Comparando-se o desvio na perda de carga total percentual nos venturis, considerando o Venturi 1 como referência, os venturis que tiveram variação de $+20 \%$, entre ângulos divergentes e convergentes, pode-se observar que todos tiveram bons resultados para baixas vazões (até $0,5 \mathrm{~m} / \mathrm{s}$ ), com V-6 (convergente) com maior queda de pressão em relação ao $\mathrm{V}-1$ controle. As figuras 3 e 4 ilustram estes resultados.

Analisando os casos em que a variação foi de $+10 \%$, o venturi em que aumentou-se somente o ângulo de convergência ( $\mathrm{V}-8)$ também foi o que mais se destacou, tanto para baixas quanto altas vazões. $\mathrm{O} V-4$, que teve acréscimos tanto no ângulo de divergência quanto de convergência, apresentou bons resultados apenas para vazões baixas. O V-12 (divergente) apresentou resultados piores para vazões de até $1 \mathrm{~m} / \mathrm{s}$, porém apresentou o melhor resultado entre os três venturis quando se trata de vazões a partir de $2 \mathrm{~m} / \mathrm{s}$.

No caso em que houve um decréscimo de 20\% nos ângulos, tanto o V-3 (divergente e convergente) quanto o V-11 (divergente) tiveram boas quedas de pressão para baixas vazões, mas apenas o segundo apresentou bons resultados também para altas vazões. É notável que o V-7 (convergente) teve resultados bastante diferentes do padrão observado na maior parte dos outros venturis.

Quando a variação foi $-10 \%$, o venturi modificado apenas no ângulo de divergência (V-13) também foi o que teve melhores resultados comparativos, embora os resultados referentes ao V-5 (convergente e divergente) sejam próximos. Novamente, o venturi convergente (V-9) foi o que apresentou as piores quedas de pressão.

$\mathrm{Na}$ comparação entre tubos de venturi que sofreram modificações apenas em seu ângulo convergente, pode-se observar que os que tiveram acréscimos (V-8 e V-6) tem resultados expressivamente melhores em relação àqueles que tiveram decréscimos $(\mathrm{V}-7 \mathrm{e} \mathrm{V}-$ 9), tanto para baixas quanto para altas vazões. 
Figura 3 - Percentual de desvio da queda de pressão quando houve variação no ângulo convergente.

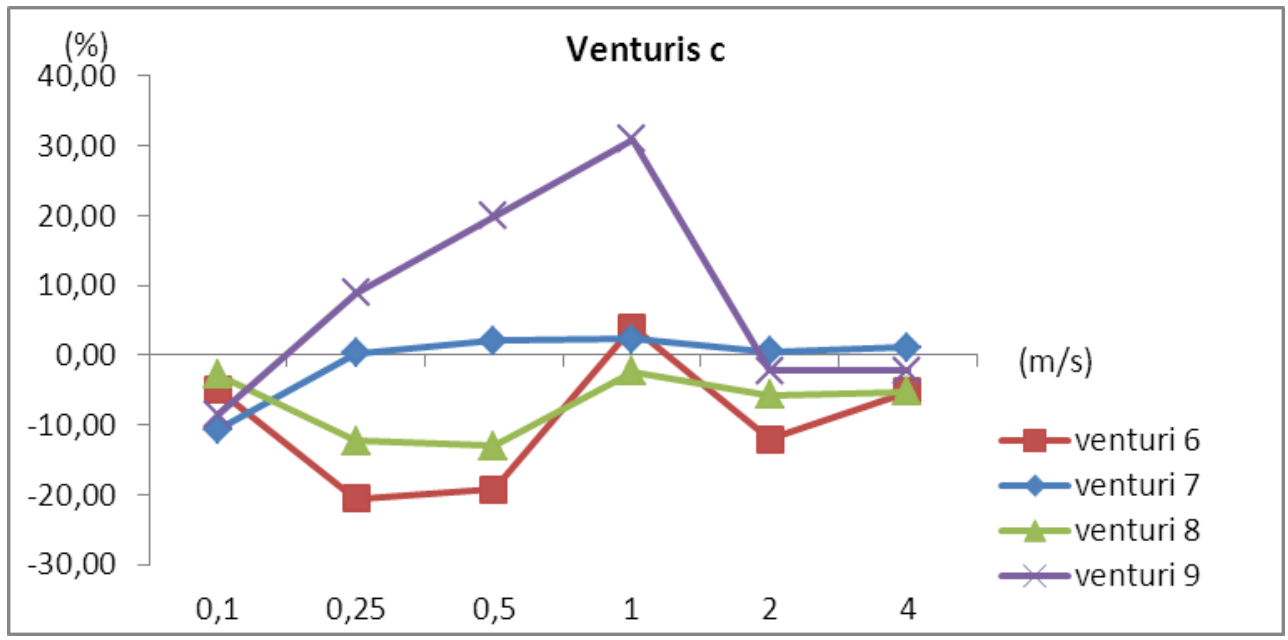

Quando essa mesma comparação é feita entre os venturis divergentes, percebe-se que apenas o V-12 não teve bons resultados para baixas vazões, embora o mesmo tenha sido bom para vazões a partir de $2 \mathrm{~m} / \mathrm{s}$. Além disso, novamente o V-11 é o que apresenta os melhores resultados, analisando de forma geral.

Na figura 4 são apresentados os resultados comparativos dos tubos de venturi que tiveram variação em seu ângulo divergente.

Figura 4 - Percentual de desvio da queda de pressão quando houve variação no ângulo divergente.

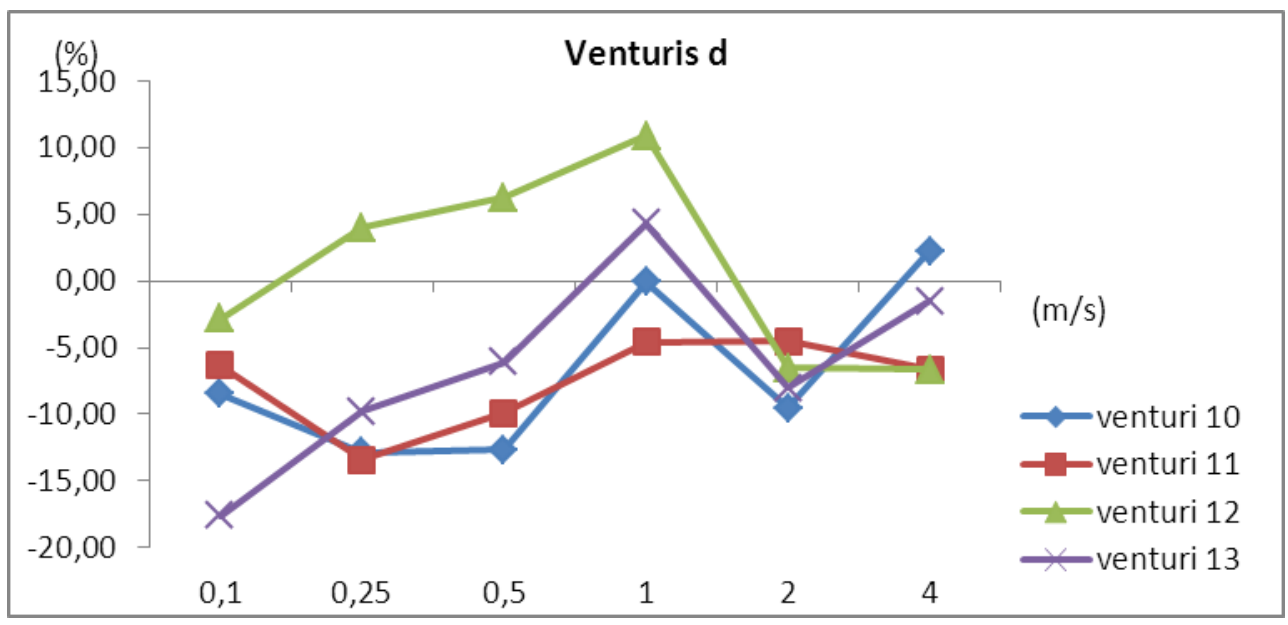

Finalmente, comparando todos os resultados obtidos, V-2, V-6 e V-8 apresentaram os melhores resultados de queda de pressão, tanto para baixas quanto para altas vazões. 
Além disso, pode-se observar que na maior parte dos casos (excluem-se apenas os V$3, \mathrm{~V}-7$ e V-11, ou seja, os que tiveram variação de $-20 \%$ ) há um pico no momento em que a velocidade é igual a $1 \mathrm{~m} / \mathrm{s}$. Outro fato notável é que as variações nas quedas de pressão tiveram melhores resultados para vazões de até $0,5 \mathrm{~m} / \mathrm{s}$, excluindo-se $\mathrm{V}-12$ e V-13 (ambos divergentes, porém $+10 \%$ e $-10 \%$, respectivamente).

De uma forma geral, na comparação entre tubos de venturi que sofreram modificações apenas em seu ângulo convergente, pode-se observar que os que tiveram acréscimos tem resultados expressivamente melhores em relação àqueles que tiveram decréscimos, tanto para baixas quanto para altas vazões. Quando essa mesma comparação é feita entre os venturis divergentes, o que teve o ângulo divergente diminuído em $20 \%$ apresentou os melhores resultados. Além disso, apenas o modelo que teve o ângulo divergente aumentado em $10 \%$ não teve bons resultados para baixas vazões.

\section{CONCLUSÃO}

Diminuir o ângulo convergente mostrou-se não ser uma boa opção, como visto nos resultados de V-7 e V-9, que foram os piores, e em V-5 e V-3, que também não tiveram bons resultados. Em contrapartida, tanto $\mathrm{V}-11$ quanto $\mathrm{V}-13$, que sofreram redução somente no ângulo divergente tiveram quedas de pressão satisfatórias. Ao mesmo tempo, o aumento dos ângulos, tanto divergente quando convergente, mostrou bons resultados, sendo V-12 a única exceção. Ainda, é necessária investigação para definir o motivo dos picos de queda de pressão quando a velocidade é igual a $1,0 \mathrm{~m} / \mathrm{s}$.

\section{REFERÊNCIAS BIBLIOGRÁFICAS}

GUERRA, V. G.; BÉTTEGA, R.; GONÇALVES, J. A. S.; COURY, J.R., Pressure Drop and Liquid Distribution in a Venturi Scrubber: Experimental Data and CFD Simulation. Ind. Eng. Chem. Res., 51 (2012), 8049-8060

HONDA, V. K.; BÉTTEGA, R.; GONÇALVES, J. A. S.; GUERRA, V. G., Estudo Numérico da Hidrodinâmica da Dispersão de Líquido no Interior de um Lavador Venturi. Cong. Bras.

Sist. Part., (2013), 76

\section{AGRADECIMENTOS}

Os autores agradecem o CNPQ - Conselho Nacional de Desenvolvimento Científico e Tecnológico pela concessão de bolsa por intermédio do programa Jovens Talentos para a Ciência. 